\title{
The prognostic significance of CMTM3 in colorectal cancer and association with the phenomenon of EMT
}

\author{
Hong Chen ${ }^{1, *}$, Rui Ren ${ }^{1, *}$, Xiaogang Zhou ${ }^{1, *}$, Daiwei Wan ${ }^{1}$, Ye Han ${ }^{1}$, Fei Liu ${ }^{2}$, Zhihua \\ $\mathrm{Xu}^{1}$, Yuting Kuang ${ }^{1}$, Hao $\mathrm{Hu}^{1}$, Yilin Wang ${ }^{3}$ and Qiaoming $\mathbf{Z h i}^{1}$ \\ ${ }^{1}$ Department of General Surgery, The First Affiliated Hospital of Soochow University, Suzhou 215006, China \\ ${ }^{2}$ Department of Gastroenterology, The First Affiliated Hospital of Soochow University, Suzhou 215006, China \\ ${ }^{3}$ Department of Hepatic Surgery, Fudan University Shanghai Cancer Center; Department of Oncology, Shanghai Medical \\ College, Fudan University, Shanghai, 200032, China \\ *These authors contributed equally to this work \\ Correspondence to: Yilin Wang, email: linglingwangyi@126.com \\ Qiaoming Zhi, email: strexboy@163.com \\ Keywords: CMTM3; colorectal cancer; prognosis; epithelial-mesenchymal transition
}

Received: May 16, $2017 \quad$ Accepted: December 13, $2017 \quad$ Published: January 02, 2018

Copyright: Chen et al. This is an open-access article distributed under the terms of the Creative Commons Attribution License 3.0 (CC BY 3.0), which permits unrestricted use, distribution, and reproduction in any medium, provided the original author and source are credited.

\section{ABSTRACT}

CKLF-like MARVEL transmembrane domain containing 3 (CMTM3), which is located at the critical tumor suppressor locus 16q22.1, has been proposed as a putative tumor suppressor gene in multiple types of malignancies. However, its role in colorectal cancer (CRC) has not been clearly defined. In this study, the potential relationships between CMTM3 and epithelial-mesenchymal transition (EMT)-related proteins were firstly analyzed in the clinical tissues. Our data demonstrated that CMTM3 presented a relatively negative staining in 130 CRC tissues, compared to their corresponding normal-appearing tissues (NATs) $(P<0.05)$. The reduction of CMTM3 protein was associated with the advanced depth of invasion, distant metastasis and tumor stage $(P<0.05)$. CRC patients with low CMTM3 levels had a poorer 5-year overall survival $(O S)$ rate $(P=0.035)$, and the decreased level of CMTM3 could be served as an independent and significant prognostic factor of CRC patients $(P=0.013)$. Meanwhile, a significant positive relationship between $C M T M-3$ and E-cadherin was found $(r=0.253, P=0.004)$, whereas CMTM3 negatively correlated with Vimentin $(r=-0.506 P=0.000)$. To better understand the tumor suppressing effect of CMTM3 in CRC progression through an EMT-dependent mechanism, we found that up-regulation of CMTM3 significantly suppressed the abilities of cell proliferation, migration and invasion in CRC cells in vitro $(P<0.05)$. The results of western blot analysis also disclosed that CMTM3 influenced the expressions of EMT-related proteins (E-cadherin, $\mathbf{N}$-cadherin and Vimentin) $(P<0.05)$. These results suggested that CMTM3 might be involved in the EMT process of CRC, and could be considered as a potentially important molecular treatment strategy for early stage CRC patients.

\section{INTRODUCTION}

Colorectal cancer (CRC) is one of the most lethal malignancies and remains one of the leading causes of cancer-related death worldwide [1]. CRC is regarded as a heterogeneous and multi-pathway disease, and the development of which is mainly associated with multiple factors, such as inflammatory bowel disease, inherited syndrome, diets with low fiber, smoke, obesity and heavy alcohol use [2]. Though great progresses in diagnostic and effective therapeutic strategies have been made, the clinical outcome of CRC is still very poor. One of the most important reasons mainly lies in local recurrences or distant metastases [3]. Clinical data indicate that approximately $30 \%$ of CRC patients will ultimately develop into distant metastases, and the 5-year overall 
survival (OS) rate of CRC patients with distant metastases is approximately $10 \%[4,5]$. Therefore, it is essential and urgent to develop novel targets (or molecules) and therapeutic methods for CRC mortality.

CKLF-like MARVEL transmembrane domain containing (CMTM) is a novel family of proteins linking chemokines and transmembrane-4 superfamily (TM4SF). In human, CMTMs are encoded by nine genes, including chemokine-like factor (CKLF) and CMTM1-8. CMTM family members have been proved to play important roles in the immune system, male reproductive system and participate in tumorigenesis [6-10]. CMTM3, which is located at the critical tumor suppressor locus 16q22.1, has been proposed as a putative tumor suppressor gene in multiple types of malignancies, such as non-small cell lung cancer [11], renal cell carcinoma [12], gastric cancer $[13,14]$, prostate cancer [15], testicular cancer [16] and hepatocellular carcinoma [17]. For example, the results of Xie's study revealed that CMTM3 was significantly down-regulated in clear cell renal cell carcinoma (ccRCC) and exerted remarkable tumor-suppressive functions in 786-0 cells [12]. In 2014, Li et al. demonstrated that CMTM3 was frequently down-regulated (or silenced) in testicular cancer cell lines and tumor tissues. Ectopic expression of CMTM3 significantly suppressed the colony formation, proliferation and migration capacity of testicular cancer cells by inducing a cell cycle arrest of G2 phase and apoptosis [16]. However, its role in the development and progression of CRC has not been clearly defined to date.

In this study, we firstly detected the protein expressions of CMTM3 and EMT-related proteins (E-cadherin and Vimentin) in $130 \mathrm{CRC}$ tissues as well as their corresponding normal-appearing tissues (NATs) by the immunohistochemistry (IHC) method. Then the potential relationships between CMTM3 expressions (or E-cadherin and Vimentin) and clinicopathological features in CRC tissues were analyzed. Kaplan-Meier survival curves were performed to evaluate the prognostic roles of CMTM3, E-cadherin and Vimentin. Spearman's rank correlation tests were used to reveal the possible associations of CMTM3 with EMT-related markers (E-cadherin and Vimentin) in CRC. Moreover, we also systematically analyzed the biological functions of CMTM3 in CRC cells after reconstitution or interference of CMTM3 expression in vitro, and the changes of EMTrelated proteins (E-cadherin, N-cadherin and Vimentin) were determined by western blot analysis.

\section{RESULTS}

\section{The expressions of CMTM3 and EMT-related proteins (E-cadherin and Vimentin) in CRC tissues}

The protein expressions of CMTM3 and EMTrelated proteins (E-cadherin and Vimentin) in $130 \mathrm{CRC}$ tissues and their corresponding NATs were determined by the immunohistochemistry (IHC) method. As shown in Figure 1A-1B, the positive staining of CMTM3 was mainly located in the cytoplasm and membrane, while E-cadherin and Vimentin proteins were located in the membrane and cytoplasm respectively (Figure $1 \mathrm{C}-1 \mathrm{~F}$ ). By calculating the relative positive percentage of CMTM3 protein between $\mathrm{CRC}$ tissues and their corresponding NATs, we found that CMTM3 presented a relative negative staining in CRC tissues $(59 / 130,45.38 \%)$, compared to NATs $(102 / 130,78.46 \%, P<0.05$, Figure $2 A)$. Similarly, of $130 \mathrm{CRC}$ samples, E-cadherin also maintained a relatively lower level in CRC samples (57/130, 43.84\%) compared to normal tissues $(118 / 130,90.77 \%, P<0.05$, Figure 2B). On the contrary, the protein expression of Vimentin in CRC tissues exhibited a higher level (85/130, $65.4 \%, P<0.05$, Figure 2C). These data suggested that aberrant expressions of CMTM3, E-cadherin and Vimentin were frequent events in CRC progressions.

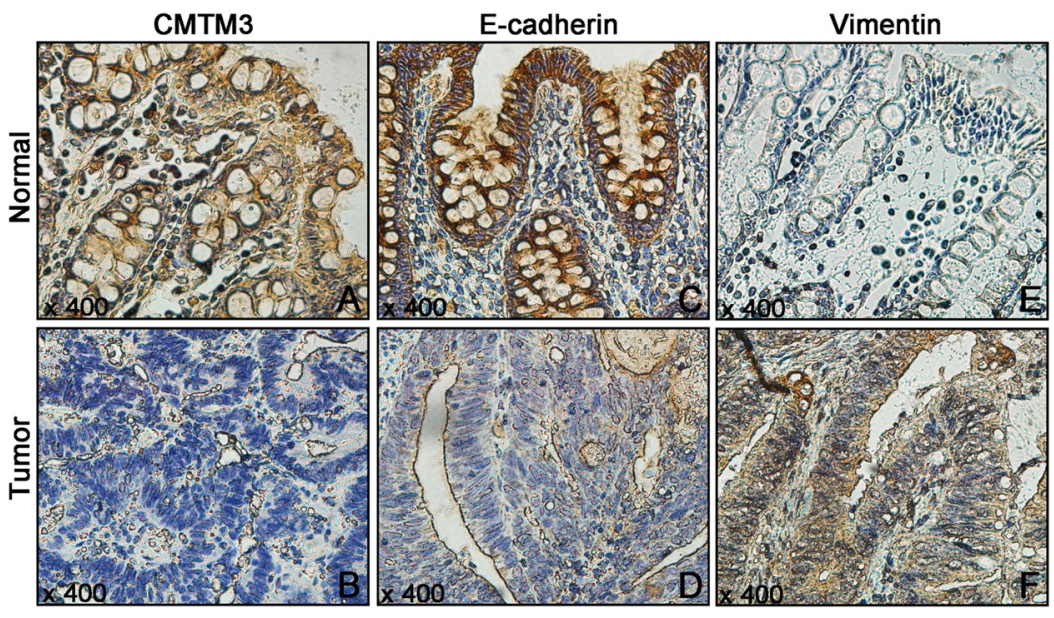

Figure 1: The protein expressions of CMTM3 and EMT-related proteins (E-cadherin and Vimentin) in $130 \mathrm{CRC}$ tissues and their corresponding NATs were detected using IHC. (A, B) CMTM3; (C, D) E-cadherin; (E, F) Vimentin. 


\section{Relationships between CMTM3 expressions (or E-cadherin and Vimentin) and clinicopathological features in CRC tissues}

To further evaluate the clinical significance of CMTM3 in CRC patients, we analyzed the potential relationships between CMTM3 expressions and clinicopathological features. Our data demonstrated that the low CMTM3 level had a positive correlation with the depth of invasion $(P=0.001)$, distant metastasis $(P=0.000)$ and TNM stage $(P=0.000)$. However, CMTM3 expressions did not correlate with the gender, age, tumor size, tumor location, tumor differentiation and lymph node metastasis (all $P>0.05$ ). Meanwhile, the importance of E-cadherin and Vimentin proteins in CRC were also assessed, and our results showed that E-cadherin was significantly associated with the depth of invasion $(P=0.013)$, lymph node metastasis $(P=0.033)$, distant metastasis $(P=0.000)$ and TNM stage $(P=0.000)$. Whereas, the Vimentin level closely correlated with the depth of invasion $(P=0.014)$, distant metastasis $(P=0.024)$ and TNM stage $(P=0.000)$ of CRC patients (Table 1).

\section{The prognostic significance of CMTM3 expressions (or E-cadherin and Vimentin) in CRC patients}

OS curves were plotted according to the expressing levels of CMTM3, E-cadherin and Vimentin by the KaplanMeier method. Our data showed that CRC patients with low CMTM3 levels had a poorer 5-year OS, compared to the patients with high CMTM3 expression $(P=0.035$, Figure 3A). Similarly, decreased expressions of E-cadherin in CRC patients also led to a poorer 5-year OS rate when compared to those patients with high E-cadherin expressions ( $P=0.001$, Figure 3B). In contrast, high Vimentin levels significantly decreased the 5-year OS rate of CRC patients $(P=0.002$, Figure 3C). Univariate analysis for OS indicated that the tumor differentiation, depth of invasion, lymph node metastasis, distant metastasis, TNM stage, CMTM3 expression, E-cadherin expression and Vimentin expression were all prognostic factors of poor prognosis (all $P<0.05$ ). Variables with a $P$ value $<0.05$ by univariate analysis were selected for the subsequent multivariate analysis using Cox's proportional hazards model. Our multivariate analysis demonstrated that three factors, including lymph node metastasis $(\mathrm{HR}=0.449$, $95 \% \mathrm{CI}=0.220-0.981, P=0.028)$, TNM stage $(\mathrm{HR}=$ $5.873,95 \% \mathrm{CI}=1.876-18.386, P=0.005)$ and CMTM3 expression $(\mathrm{HR}=2.579,95 \% \mathrm{CI}=1.219-5.456, P=$ 0.013 ), retained as independent and significant prognostic factors for survival in CRC (Table 2).

\section{Relationships between CMTM3 and EMT- related proteins (E-cadherin and Vimentin) in CRC tissues}

The possible relationships between CMTM3 and EMT-related proteins (E-cadherin and Vimentin) in 130 CRC samples were evaluated using SPSS software, and a significant positive correlation of CMTM-3 with E-cadherin was found ( $r=0.253, P=0.004$ ) in CRC patients, while the protein expressions of CMTM3 negatively correlated with the Vimentin levels $(r=$ $-0.506 P=0.000$, Table 3 ). These results were basically consistent with the IHC staining of CMTM3, E-cadherin and Vimentin in CRC tissues, which implied that CMTM3 might be involved in the EMT process of CRC.

\section{Effects of CMTM3 on cell proliferation, migration and invasion in CRC cells}

To analyze the effects of CMTM3 on CRC progression in vitro, we obtained CMTM3 up- and downregulating cells for our subsequent experiments in vitro. Our data of PCR analysis confirmed the transfection efficiency of CMTM3 plasmids or siRNAs $(P<0.05$, Figure $4 \mathrm{~A}-4 \mathrm{C})$. The results of $\mathrm{CCK}-8$ showed that CMTM3 enhanced the abilities of cell proliferation at 72 or 96 h in SW1116 cells, whereas transfection with siRNA-CMTM3 in SW620 cells significantly inhibited the cell proliferation at 48,72 , or $96 \mathrm{~h}$, compared to the siRNA-Con group $(P<0.05$, Figure 4D-4E). Meanwhile, the abilities of tumor metastasis were evaluated by cell migration and invasion assays. As shown in Figure 5A$5 \mathrm{D}$, up-regulation of CMTM3 significantly suppressed the cell migration and invasion abilities of SW1116 cells, compared to the empty vector group. Oppositely,
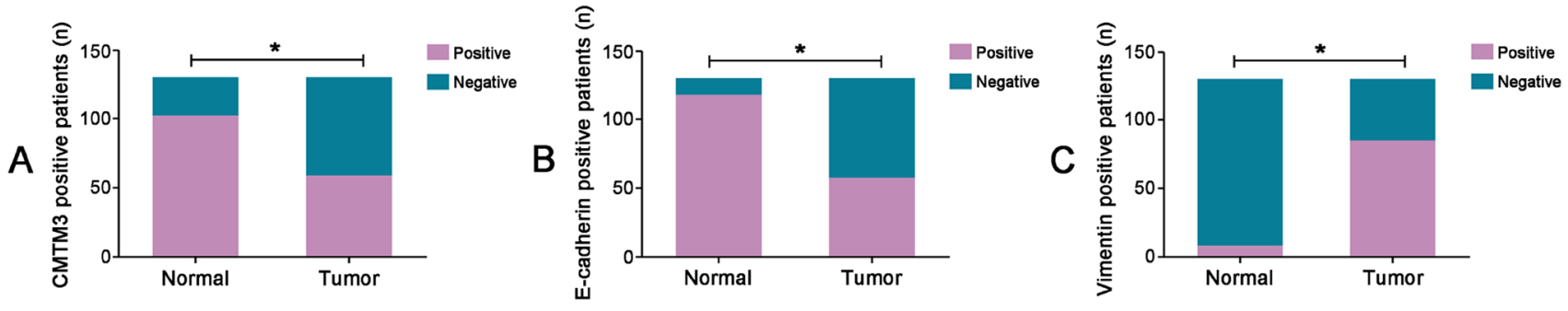

Figure 2: The bar charts represented the number of CMTM3 and EMT-related protein (E-cadherin and Vimentin) positive staining in 130 CRC tissues and their corresponding NATs. (A) CMTM3; (B) E-cadherin; (C) Vimentin. $\left({ }^{*} P<0.05\right)$ 
Table 1: Relationships between CMTM3 expressions (or EMT-related proteins) and clinicopathological features in CRC tissues $\left({ }^{*} P<0.05\right)$

\begin{tabular}{|c|c|c|c|c|c|c|c|c|c|c|}
\hline \multirow{2}{*}{ Parameters } & \multirow{2}{*}{$n$} & \multicolumn{3}{|c|}{ СМТМ3 } & \multicolumn{3}{|c|}{ E-cadherin } & \multicolumn{3}{|c|}{ Vimentin } \\
\hline & & Low & High & $P$ & Low & High & $P$ & Low & High & $P$ \\
\hline \multicolumn{11}{|l|}{ Gender } \\
\hline Male & 69 & 39 & 30 & 0.642 & 42 & 27 & 0.249 & 25 & 44 & 0.680 \\
\hline Female & 61 & 32 & 29 & & 31 & 30 & & 20 & 41 & \\
\hline \multicolumn{11}{|l|}{ Age } \\
\hline$\leq 60$ & 62 & 38 & 24 & 0.144 & 38 & 24 & 0.260 & 18 & 44 & 0.201 \\
\hline$>60$ & 68 & 33 & 35 & & 35 & 33 & & 27 & 41 & \\
\hline \multicolumn{11}{|l|}{ Tumor size } \\
\hline$\leq 5 \mathrm{~cm}$ & 88 & 50 & 38 & 0.465 & 49 & 39 & 0.875 & 28 & 60 & 0.332 \\
\hline$>5 \mathrm{~cm}$ & 42 & 21 & 21 & & 24 & 18 & & 17 & 25 & \\
\hline \multicolumn{11}{|l|}{ Tumor location } \\
\hline Colon & 72 & 36 & 36 & 0.239 & 37 & 35 & 0.222 & 24 & 48 & 0.732 \\
\hline Rectum & 58 & 35 & 23 & & 36 & 22 & & 21 & 37 & \\
\hline \multicolumn{11}{|l|}{$\begin{array}{l}\text { Tumor } \\
\text { differentiation }\end{array}$} \\
\hline Well & 11 & 6 & 5 & 0.245 & 5 & 6 & 0.751 & 6 & 5 & 0.291 \\
\hline Moderately & 81 & 40 & 41 & & 46 & 35 & & 28 & 53 & \\
\hline Poorly & 38 & 25 & 13 & & 22 & 16 & & 11 & 27 & \\
\hline \multicolumn{11}{|c|}{ Depth of invasion } \\
\hline $\mathrm{T} 1-\mathrm{T} 2$ & 40 & 13 & 27 & $0.001^{*}$ & 16 & 24 & $0.013^{*}$ & 20 & 20 & $0.014^{*}$ \\
\hline T3-T4 & 90 & 58 & 32 & & 57 & 33 & & 25 & 65 & \\
\hline \multicolumn{11}{|l|}{$\begin{array}{l}\text { Lymph node } \\
\text { metastasis }\end{array}$} \\
\hline No & 73 & 35 & 38 & 0.084 & 35 & 38 & $0.033^{*}$ & 25 & 48 & 0.920 \\
\hline Yes & 57 & 36 & 21 & & 38 & 19 & & 20 & 37 & \\
\hline \multicolumn{11}{|c|}{ Distant Metastasis } \\
\hline M0 & 93 & 41 & 52 & $0.000^{*}$ & 42 & 51 & $0.000^{*}$ & 38 & 55 & $0.024^{*}$ \\
\hline M1 & 37 & 30 & 7 & & 31 & 6 & & 7 & 30 & \\
\hline \multicolumn{11}{|l|}{ TNM stage } \\
\hline I-II & 67 & 20 & 47 & $0.000^{*}$ & 30 & 37 & $0.000^{*}$ & 34 & 33 & $0.000^{*}$ \\
\hline III-IV & 63 & 51 & 12 & & 43 & 20 & & 11 & 52 & \\
\hline
\end{tabular}
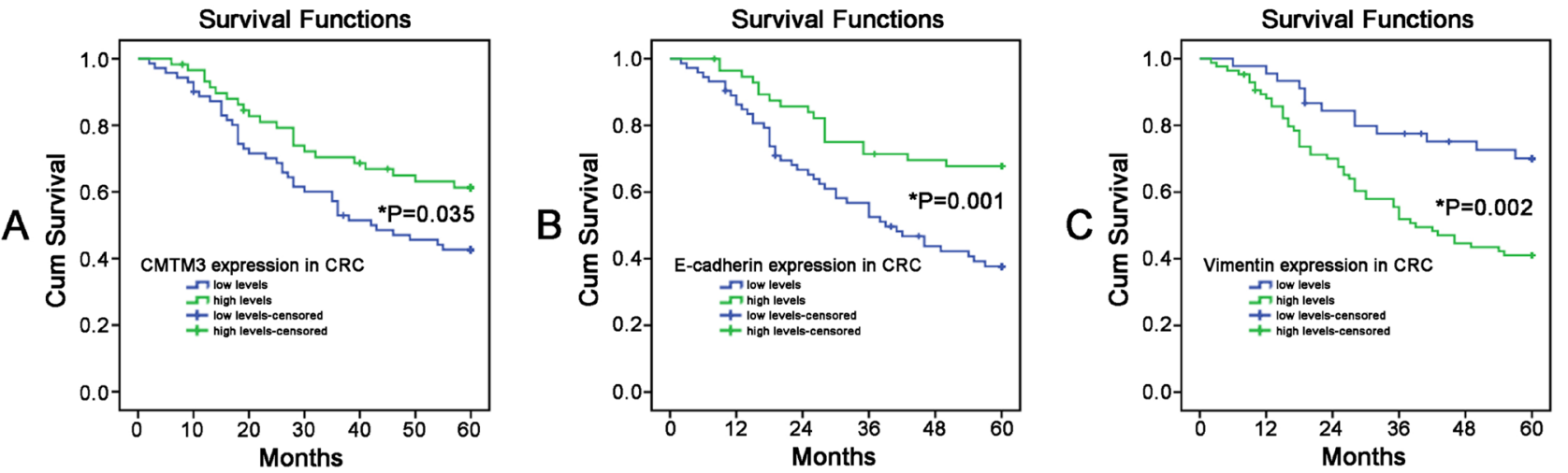

Figure 3: The Kaplan-Meier survival curves were performed to predict the 5-year survival rates of CRC patients. (A) CMTM3; (B) E-cadherin; (C) Vimentin. $\left({ }^{*} P<0.05\right)$ 
Table 2: Univariate and multivariate analyses of OS (Cox's regression model)

\begin{tabular}{lcccccc}
\hline \multirow{2}{*}{ Variables } & \multicolumn{3}{c}{ Univariate analysis } & \multicolumn{3}{c}{ Multivariate analysis } \\
\cline { 2 - 7 } & HR & $\mathbf{9 5 \%}$ CI & $\boldsymbol{P}$ & HR & $\mathbf{9 5 \% ~ C I ~}$ & $\boldsymbol{P}$ \\
\hline Gender (Male/Female) & 1.744 & $0.643-1.744$ & 0.822 & - & - & - \\
Age $(\leq 60 />60)$ & 0.632 & $0.538-1.457$ & 0.886 & - & - & - \\
Tumor size ( $\leq 5 />5)$ & 1.057 & $0.625-1.789$ & 0.835 & - & - & - \\
Tumor location (Colon/Rectum) & 1.058 & $0.641-1.744$ & 0.826 & - & - & - \\
Tumor differentiation & 2.843 & $1.807-4.472$ & $0.000^{*}$ & 0.156 & $0.020-1.195$ & 0.074 \\
(Well/Moderately/Poorly) & & & & & & \\
Depth of invasion (T1-T2/ T3-T4) & 2.743 & $1.392-5.404$ & $0.004^{*}$ & 1.874 & $0.903-3.891$ & 0.092 \\
Lymph node metastasis (No/Yes) & 1.326 & $0.823-2.626$ & $0.018^{*}$ & 0.449 & $0.220-0.981$ & $0.028^{*}$ \\
Distant Metastasis (M0/M1) & 3.948 & $2.384-6.538$ & $0.000^{*}$ & 1.469 & $0.725-2.977$ & 0.286 \\
TNM stage (I-II/ III-IV) & 4.078 & $2.236-7.151$ & $0.000^{*}$ & 5.873 & $1.876-18.386$ & $0.005^{*}$ \\
CMTM3 expression (Low/High) & 0.577 & $0.343-0.971$ & $0.038^{*}$ & 2.579 & $1.219-5.456$ & $0.013^{*}$ \\
E-cadherin expression (Low/High) & 0.417 & $0.241-0.723$ & $0.002^{*}$ & 1.053 & $0.536-2.066$ & 0.881 \\
Vimentin expression (Low/High) & 2.502 & $1.356-4.615$ & $0.003^{*}$ & 1.685 & $0.815-3.487$ & 0.159 \\
\hline
\end{tabular}

HR Hazard ratio, CI Confidence interval, ${ }^{*} P<0.05$.

interfering CMTM3 expression could increase the number of migrated or invaded SW620 cells $(P<0.05)$.

\section{CMTM3 inhibited malignant transformation through EMT in CRC cells}

Since we proved that CMTM3 predominantly correlated with the EMT phenotype in clinical tissues, we also wondered whether aberrant CMTM3 expression could influence the protein levels of EMT-related markers (E-cadherin, N-cadherin and Viementin) in vitro. The results of western blot analysis revealed that CMTM3 over-expression increased the E-cadherin expression, and decreased the levels of $\mathrm{N}$-cadherin and Vimentin in SW1116 cells. Opposite phenomena were observed when CMTM3 was effectively interfered in SW620 cells (Figure 6A-6D). Our data strongly implied that CMTM3 exhibited the inhibitory effects of EMT in CRC cells.

\section{DISCUSSION}

Despite recent improvements in molecular diagnostics, gene sequencing and targeted therapies, the prognosis of patients with CRC is still unsatisfactory.

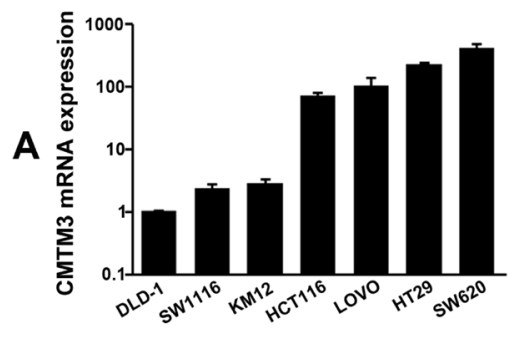

B
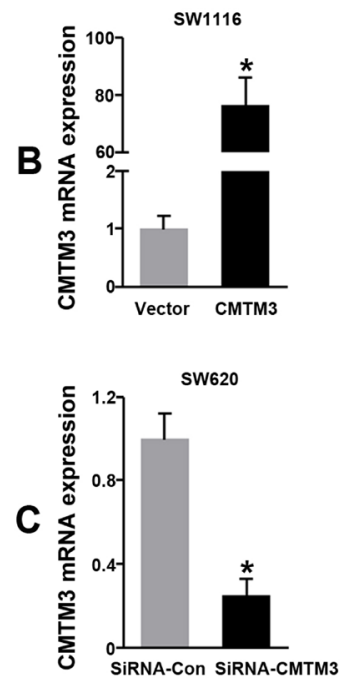
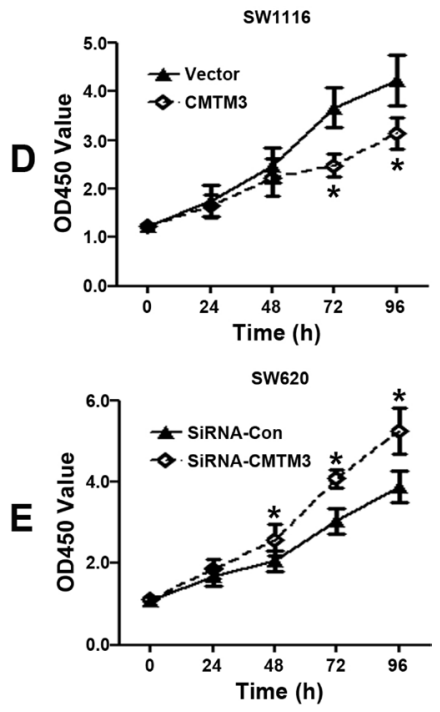

Figure 4: Effects of CMTM3 on cell proliferation in CRC cells. (A) CMTM3 mRNA expressions in different CRC cell lines; (B-C) The transfection efficiencies of CMTM3 plasmids or siRNAs were confirmed by qRT-PCR; (D-E) Effects of CMTM3 on cell proliferation in $\mathrm{CRC}$ cells were determined by the CCK-8 assay. $\left({ }^{*} P<0.05\right)$ 
Table 3: Relationships between the expressions of CMTM3 and EMT-related proteins $(* P<0.05)$

\begin{tabular}{lcccc}
\hline & \multicolumn{2}{c}{ CMTM3 } & & \multicolumn{1}{c}{ Low } \\
\cline { 2 - 4 } & High & $\boldsymbol{r}$ & 0.004 \\
\hline E-cadherin & 34 & 23 & 0.253 & \\
High & 25 & 48 & & \\
Low & & & & \\
Vimentin & 23 & 62 & -0.506 & \\
High & 36 & 9 & & \\
Low & & 9000 \\
\hline
\end{tabular}

CRC remains a highly lethal disease. The main cause of death in CRC patients is tumor progression with metastasis. Emerging available literatures indicate that epithelial-mesenchymal transition is a crucial event in tumor progression, which causes an increase of tumor cells with more invasive and metastatic properties $[18,19]$. The phenomenon of EMT was firstly proposed by Garry Greenburg and Hay in 1982, and now it is widely accepted that in epithelial cancers, including colorectal cancer. EMT is associated with the three major steps of cancer development: invasion, dissemination and metastasis. EMT is a developmental program that enables stationary epithelial cells to gain the ability to migrate and invade as single cells [19,20]. Tumor cells reactivate EMT to acquire molecular alterations that enable the partial loss of epithelial features and partial gain of a mesenchymal phenotype. A variety of markers have been used to describe EMT. E-cadherin, intergrins and cytokeratins are the most commonly used epithelial markers, and $\mathrm{N}$-cadherin, Vimentin or fibronectin are for the mesenchymal [21-23]. In this study, we detected the protein expressions of E-cadherin and Vimentin in 130 CRC tissues using IHC, and our results re-confirmed the dynamic process of EMT in CRC with the downregulation of E-cadherin and up-regulation of Vimentin. Our data also demonstrated that loss of E-cadherin was associated with some metastasis-related clinicopathogical parameters, such as the depth of invasion, lymph node metastasis, distant metastasis and TNM stage, whereas high Vimentin closely correlated with these three factors. OS curves were plotted according to the expressing levels of E-cadherin and Vimentin by the Kaplan-Meier method. Our results disclosed that CRC patients with low E-cadherin levels had a poorer 5-year OS rate, while loss of Vimentin could lead to an improved clinical outcome in CRC. All these results were consistent with the predictions of previous studies, which supported the role of EMT in $\mathrm{CRC}$ progression, particularly the changes of epithelial or mesenchymal traits during EMT [24, 25].

CMTM proteins contain a MARVEL domain, which is involved in many essential cellular processes including tumor suppression. Recently, the abnormal expressions of CMTM3 were reported in several malignant tumors [11-17]. For example, Su et al. found that the expression of CMTM3 was remarkably weaker in gastric cancer tissues than in normal mucosae. Interestingly, CMTM3
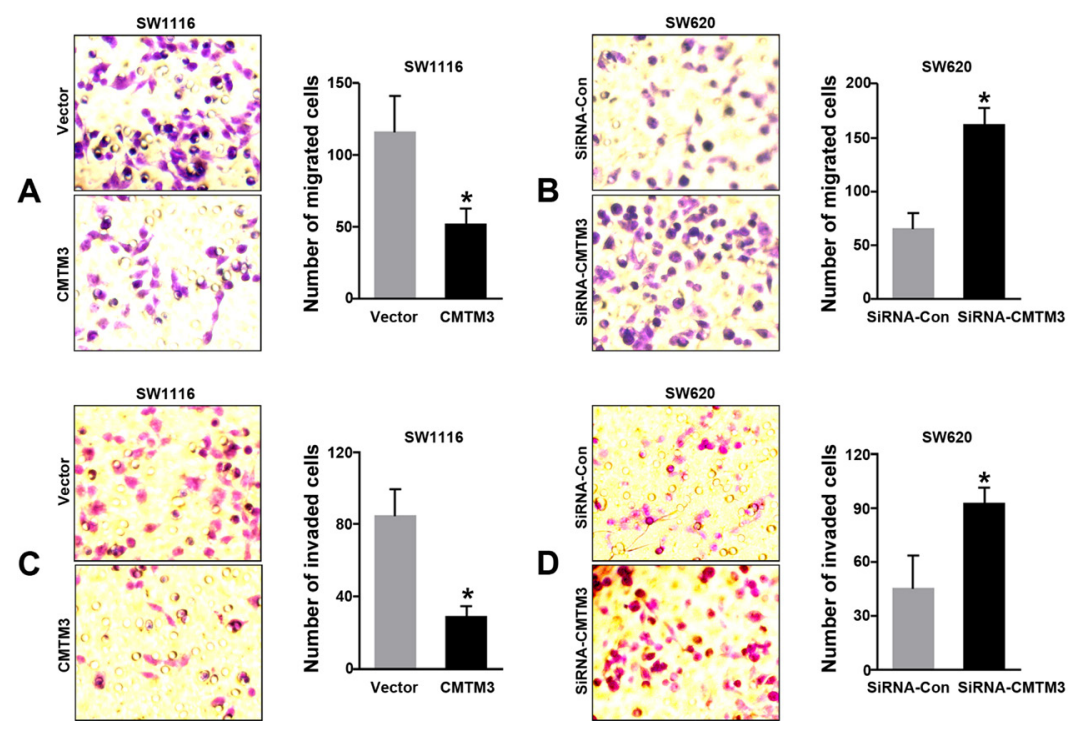

Figure 5: Effects of CMTM3 on cell migration and invasion in CRC cells. (A-B) Effects of CMTM3 on cell migration in CRC cells were observed and calculated under the microscope; (C-D) Effects of CMTM3 on cell invasion in CRC cells. $\left({ }^{*} P<0.05\right)$ 
expression was also associated with the prognosis of gastric cancer patients [13]. In 2015, Hu's study observed that ectopic expression of CMTM3 in LNCaP cells led to a significant inhibition of cell proliferation, migration and invasion, and Erk1/2 activity as p-Erk1/2 was remarkably reduced when CMTM3 was over-expressed [15]. These findings all proved that CMTM3 had a suppressive function in tumor progression. Only one study presented the oncogenic effects of CMTM3 in glioblastoma by Delic, in which CMTM1, 3, and 7 were proved to promote the abilities of tumor cell invasion, and higher CMTM1 and 3 expressions significantly correlated with shorter overall survivals [26]. To further explore the potential tumor-suppressive mechanisms of CMTM3 in cancers, several studies were performed and concluded as follows: (i) the silence of CMTM3 was due to aberrant promoter $\mathrm{CpG}$ methylation that could be reversed by pharmacologic demethylation [16, 27-29]; (ii) the expression of MMP2 and the phosphorylation of Erk1/2 were decreased when CMTM3 was restored [13, 15]; (iii) CMTM3 induced a cell cycle arrest of G2 phase and apoptosis by upregulating the expression of p21 and increasing the cleavage of caspase 9, 8, 3 and PARP [16]; (iv) CMTM3 inhibited EMT through the STAT3/Twist1/EMT or JAK2/ STAT3 signaling pathway $[14,17]$. Moreover, Yuan et al. implied that CMTM3 could decrease EGFR expression, facilitate EGFR degradation, inhibit the EGF-mediated tumorigenicity of tumor cells and influence the STAT3 signaling [30]. All these above evidence could effectively clarify the mechanisms of CMTM3 in cancers, but its clinical significance in CRC was still unknown.

Our subsequent experiments analyzed the CMTM3 expressions in $130 \mathrm{CRC}$ tissues and their corresponding NATs by IHC. The results showed that CMTM3 presented a relative negative staining in CRC tissues, which was similar to the E-cadherin expression. CMTM3 had a negative correlation with some specific metastasis-related features, such as the depth of invasion, distant metastasis and TNM stage. OS curves were plotted according to the expressing levels of CMTM3, and the data proposed that CRC patients with low CMTM3 levels had a poorer 5-year OS, compared to patients with high CMTM3. Moreover, Cox's proportional hazards model also indicated that down-expression of CMTM3 could serve as an independent and significant prognostic factor for CRC survival, similar to lymph node metastasis or TNM stage. All these results demonstrated that CMTM3 played a tumor suppressive role in $\mathrm{CRC}$, and might be served as a prognostic biomarker for CRC patients. To better reveal the possible relationships between CMTM3 and EMT, the Spearman's rank correlation test was performed. A significant positive correlation between CMTM-3 and E-cadherin was found in CRC patients, whereas CMTM3 negatively correlated with Vimentin.

We also confirmed CMTM3 as a tumor suppressor gene in CRC cells. Our experiments in vitro implied that up-regulation of CMTM3 suppressed the abilities of cell proliferation, migration and invasion in SW1116 cells. While silencing CMTM3 by siRNAs exhibited a promoting effect of tumorigenesis in SW620 cells. Since we proved that CMTM3 predominantly correlated with the EMT phenotype in clinical tissues, we speculated that CMTM3 might also inhibit the CRC progression through an EMT-dependent mechanism. Fortunately, our results of western blot analysis demonstrated that CMTM3 influenced the expressions of EMT-related proteins, and exhibited an inhibitory effect of EMT in CRC cells.
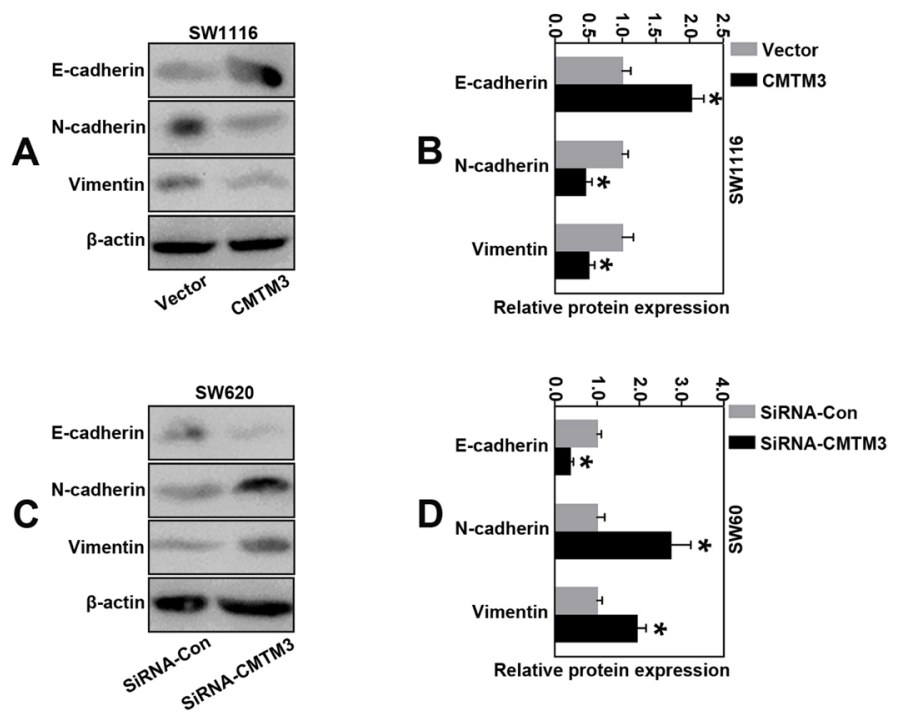

Figure 6: CMTM3 inhibited malignant transformation through EMT in CRC cells. (A-B) The expressions of EMT-related proteins (E-cadherin, N-cadherin and Vimentin) in CMTM3-overexpressing SW1116 cells were determined by western blot analysis; (C-D) The expressions of EMT-related proteins (E-cadherin, N-cadherin and Vimentin) in CMTM3-downregulating SW620 cells were determined by western blot analysis. $\left({ }^{*} P<0.05\right)$. 
Though more investigations are warranted to interpret the possible mechanisms of CMTM3, our study is the first one to evaluate its clinical significance in CRC. Our data disclosed that CMTM3 presented a relatively negative staining in $\mathrm{CRC}$ tissues, compared to their corresponding NATs. The reduction of CMTM3 protein was associated with advanced depth of invasion, distant metastasis and tumor stage. CRC patients with low CMTM3 levels had a poorer 5-year OS rate, and the down-expressing level of CMTM3 could be served as an independent and significant prognostic factor for CRC survival. Besides, a significant positive relationship between CMTM3 and E-cadherin was found, and CMTM3 negatively correlated with Vimentin. The results in vitro also confirmed that CMTM3 could influence the expressions of EMT-related proteins, and exhibited an inhibitory effect of growth and metastasis in CRC cells. These results suggested that CMTM3 might be involved in the EMT process of CRC, and up-regulation of CMTM3 could be considered as a potentially important molecular treatment strategy for early stage CRC patients.

\section{MATERIALS AND METHODS}

\section{Patients and tissue samples}

Surgical specimens of CRC tissues and their corresponding NATs (which were $5 \mathrm{~cm}$ away from the edge of tumors) were recruited from 130 patients from the Department of General Surgery, the First Affiliated Hospital of Soochow University from Jan 2008 to Dec 2010. Formalin-fixed tumor tissues were used for the IHC staining including tumor samples and matched adjacent non-tumor tissues. Detailed clinicopathological information were recorded, such as the gender, age, tumor size, tumor location, tumor differentiation, depth of invasion, lymph node metastasis, distant metastasis and TNM stage (I-IV) according to the American Joint Committee on Cancer (AJCC) TNM staging system. None of the patients had been received chemotherapy or radiation therapy before operations. All patients in this study entered a follow-up program and were closely followed every 3-6 months. All samples were obtained following patient consents and approved by the Ethics Committee of the First Affiliated Hospital of Soochow University. CRC cell lines (DLD-1, SW1116, KM12, HCT116, LOVO, HT29 and SW620) were purchased from ATCC (American Type Culture Collection) (Manassas, VA, USA) and routinely cultured in RPMI-1640 culture medium supplemented with $10 \%$ fetal bovine serum (FBS) in a humidified cell incubator with an atmosphere of $5 \% \mathrm{CO} 2$ at $37^{\circ} \mathrm{C}$.

\section{Immunohistochemical (IHC) Staining}

Tissues were fixed in neutral buffered formalin and embedded in paraffin. The sections of $4 \mu \mathrm{m}$ thickness were cut and placed on silane-coated slides. The corresponding sections were also stained with $\mathrm{H} \& \mathrm{E}$ and microscopically examined to confirm the diagnosis of CRC by two experienced pathologists. The paraffin sections were dewaxed using xylene, followed by rehydration using gradient ethanol. Antigen retrieval was performed by pretreatment of the slides in $10 \mathrm{mmol} / \mathrm{L}$ citrate buffer ( $\mathrm{pH}$ 6.0) for $15 \mathrm{~min}$ at $95^{\circ} \mathrm{C}$ using a microwave oven. Endogenous peroxidase was blocked by hydrogen peroxide (3\%) for $10 \mathrm{~min}$. After washing with $10 \mathrm{mM}$ $1 \times$ phosphate-buffered saline (PBS, $\mathrm{pH} 7.4)$, the slides were blocked with $5 \%$ normal blocking serum for $30 \mathrm{~min}$ at room temperature. Subsequently, IHC staining was performed separately overnight at $4^{\circ} \mathrm{C}$ with primary antiCMTM3 (rabbit polyclonal to CMTM3, 1:30, Abcam, USA), anti-Ecadherin (mouse monoclonal to E-cadherin, 1:300, Immunoway, USA) and anti-Vimentin (rabbit polyclonal to Vimentin, 1:300, Immunoway, USA). After washing with 1x PBS, samples were treated with biotinylated secondary antibody, and then visualized with diaminobenzidine. Finally, the sections were counterstained with haematoxylin.

\section{Evaluation of IHC staining density}

The staining intensities of CMTM3, E-cadherin and Vimentin were quantified by two independent experienced pathologists who were blinded to the data of patients. The number of positive tumor cells and degrees of staining were recorded in 5 randomly chosen fields. The extent of positive tumor cells was quantified according to the following criteria: score 0 ( $<10 \%$ positively stained cells), score 1 (11-25\% positively stained cells), score 2 (26-50 $\%$ positively stained cells), score 3 ( $>51-75 \%$ positively stained cells) and score 4 (>75\% positively stained cells). Staining intensity was scored as follows: $0=$ negative, $1=$ weakly positive, 2 = moderately positive and $3=$ strongly positive. Finally the above scores were multiplied to obtain a total score: $<6$ as a low level (or negative staining) and $\geq 6$ as the high level (or positive staining).

\section{Quantitative reverse transcription-PCR (qRT- PCR)}

Total RNA was extracted from cells using Trizol solution (Invitrogen, Carlsbad, CA, USA). Reverse transcription (RT) was performed in a $20-\mu$ l reaction according to the manufacturer's recommendations (Qiagen Inc., Valencia, CA, USA). qRT-PCR analyses were performed using the CMTM3 (Forward: 5'-CGAGTCGGGTCTCTCATTCAT-3' and Reverse: 5'-CCCTGCCACTTGTCATTCAG-3') and GAPDH (Forward: 5'-GGAGCGAGATCCCTCCAAAAT-3' and Reverse: 5'-GGCTGTTGTCATACTTCTCATGG-3') primers. GAPDH was used as a reference to obtain the relative fold changes for targets using the comparative $\mathrm{Ct}$ method. Each sample was analyzed using the ABI Prism 7000 (Applied Biosystems, Foster City, USA) in triplicate. 


\section{CMTM3 over-expressing plasmid construction and small interfering RNA}

The full-length cDNA of CMTM3 was amplified by the following primers: 5'-CCGCT CGAGATGTGGCCCCCAGACC-3' (F) and 5'-CGCGG ATCCTCAGTCAGAGTCCGAGTCG-3' (R). The products were subsequently cloned into the pcDNA3.1 vector using the XhoI and BamH1 restriction sites. Sequence fidelity and reading frame accuracy of CMTM3-overexpressing plasmid were verified by DNA sequencing analysis. Plasmid for transfection was prepared using an EndoFree Plasmid Maxi Kit (Qiagen, Tokyo, Japan). For CMTM3 small interfering RNA assay, the sequences of SiRNA-CMTM3 were UAAAAGUGAUGAAUGAGAGAC (Sense) and CUCUCAUUCAUCACUUUUAUC (Antisense). Nonspecific sequences were used as a negative control (NC). After $48 \mathrm{~h}$ transfection with Lipofectamine 2000 in accordance with the manufacturer's procedure (Invitrogen, CA, USA), cells were collected. The transfection efficiencies of over-expressing plasmids or siRNAs were detected by qRT-PCR.

\section{CCK-8 cell proliferation assay}

Cell Counting Kit-8 (CCK-8) (Dojindo Laboratories, Japan) was used to measure the cell proliferative rates of CRC cells. After 24-h transfection with CMTM3 overexpressing vectors or SiRNAs, cells were digested and seeded into 96 -well plates $\left(1 \times 10^{3}\right.$ cells per well). The CCK-8 assay was performed on day $0,1,2,3$ and 4 respectively. $10 \mu \mathrm{l}$ of CCK- 8 was added to each well and the plate was incubated for $2 \mathrm{~h}$ at $37^{\circ} \mathrm{C}$. At the endpoint of incubation, the absorbance was measured at $450 \mathrm{~nm}$ using a Vmax microplate spectrophotometer (Molecular Devices, Sunnyvale, CA). Each sample was assayed in triplicate.

\section{Cell migration and invasion assay}

To measure cell migration or invasion, $8-\mu \mathrm{m}$ pore size transwell chambers (24-well format, Corning, NY, USA) were used. For migration assay, after $24 \mathrm{~h}$ transfection, cells were harvested and seeded (a density of $1 \times 10^{4}$ per well) in the upper chamber with $200 \mu \mathrm{l}$ serum-free medium, while $600 \mu \mathrm{l}$ culture medium with $10 \%$ FBS was placed in the lower chamber. After $48 \mathrm{~h}$ incubation at $37^{\circ} \mathrm{C}$, the non-migrated cells were removed with cotton swabs. Finally, the insert membranes were fixed in $3.7 \%$ methanol for $10 \mathrm{~min}$ and stained with $0.1 \%$ crystal violet for $20 \mathrm{~min}$. The permeating cells were counted and photographed under the inverted light System Microscope IX71 (Olympus, Japan). For the cell invasion study, the inserts of chamber were prepared by coating the upper surfaces with Matrigel (BD Biosciences, CA, USA).
Other procedures were the same with the migration assay as described above.

\section{Western blotting analysis}

Firstly, cells were harvested and lysed in RIPA buffer (50 mM Tris- $\mathrm{HCl}$ [pH 7.4], $150 \mathrm{mM} \mathrm{NaCl}, 1 \%$ sodium deoxycholate, $0.1 \%$ sodium dodecyl sulfate, $1 \%$ NP-40, $1 \mathrm{mM}$ EDTA and $1 \mathrm{mM}$ PMSF). The protein concentration of the cell lysates was quantified using a BCA Protein Assay Kit (Pierce). Proteins were separated by SDS-polyacrylamide gel electrophoresis (SDS-PAGE) and subsequently transferred to PVDF membranes (NENTM Life Science Products, Boston, MA, USA). The blots were blocked with 5\% non-fat milk in TBST saline at room temperature for $1 \mathrm{~h}$ and incubated with the appropriate primary antibodies for CMTM3 (rabbit polyclonal to CMTM3, 1:500, Abcam, USA), E-cadherin (mouse monoclonal to E-cadherin, 1:1000, Immunoway, USA), N-catenin (mouse monoclonal to Vimentin, 1:1000, Immunoway, USA) or Vimentin (rabbit polyclonal to Vimentin, 1:300, Immunoway, USA) at $4^{\circ} \mathrm{C}$ overnight. The membranes were then incubated with anti-rabbit or anti-mouse secondary antibodies for $2 \mathrm{~h}$ before development using a chemiluminescence substrate (Millipore, Billerica, MA, USA). Protein levels were normalized to $\beta$-actin using a polyclonal anti- $\beta$-actin antibody (Abcam, 1:1000, Abcam, USA).

\section{Statistical analysis}

The relationships between CMTM3/E-cadherin/ Vimentin expressions and clinicopathological features were investigated using chi-square test or Fisher's exact test. Spearman's rank correlation test was performed to analysis the correlations of CMTM3 with E-cadherin (or Vimentin). OS was defined as the intervals form the dates of operations to dates of death (or the last known follow up). Survival curves were calculated by the Kaplan-Meier method, and the differences among different groups were analyzed by the log-rank test. For univariate and multivariate analyses, the Cox proportional hazards model was used to determine the prognostic significance. Differences between groups were analyzed using the Student $t$-test or ANOVA. P value less than 0.05 was validated as the statistical significance. All statistical analyses were performed with SPSS 19.0 software (SPSS Inc., Chicago, IL, USA).

\section{CONFLICTS OF INTEREST}

The authors report no conflicts of interest with this study.

\section{FUNDING}

This study was supported by grants from the Special Subject of Diagnosis Treatment of Key Clinical Diseases 
of Shuzhou City Sci-tech Bureau (LCZX201401), and the Project of Invigorating Health Care through Science, Technology and Education, Jiangsu Provincial Medical Youth Talent (QNRC2016723).

\section{REFERENCES}

1. Siegel R, Naishadham D, Jemal A. Cancer statistics, 2013. CA Cancer J Clin. 2013; 63:11-30.

2. Harpaz N, Polydorides AD. Colorectal dysplasia in chronic inflammatory bowel disease: pathology, clinical implications, and pathogenesis. Arch Pathol Lab Med. 2010; 134:876-895.

3. Chua YJ, Zalcberg JR. Progress and challenges in the adjuvant treatment of stage II and III colon cancers. Expert Rev Anticancer Ther. 2008; 8:595-604.

4. Bilchik AJ, DiNome M, Saha S, Turner RR, Wiese D, McCarter M, Hoon DS, Morton DL. Prospective multicenter trial of staging adequacy in colon cancer: preliminary results. Arch Surg. 2006; 141:527-533.

5. Patel A, Sun W. Ziv-aflibercept in metastatic colorectal cancer. Biologics. 2014; 8:13-25.

6. Han W, Ding P, Xu M, Wang L, Rui M, Shi S, Liu Y, Zheng Y, Chen Y, Yang T, Ma D. Identification of eight genes encoding chemokine-like factor superfamily members 1-8 (CKLFSF1-8) by in silico cloning and experimental validation. Genomics. 2003; 81:609-617.

7. Song HS, Shi S, Lu XZ, Gao F, Yan L, Wang Y, Zhuang H. Intracellular CMTM2 negatively regulates human immunodeficiency virus type-1 transcription through targeting the transcription factors AP-1 and CREB. Chin Med J. 2010; 123:2440-2445.

8. Plate M, Li T, Wang Y, Mo X, Zhang Y, Ma D, Han W. Identification and characterization of CMTM4, a novel gene with inhibitory effects on HeLa cell growth through Inducing G2/M phase accumulation. Mol Cells. 2010; 29:355-361.

9. Niu J, Li H, Zhang Y, Li J, Xie M, Li L, Qin X, Qin Y, Guo X, Jiang Q, Liu Y, Chen S, Huang X, et al. Aberrant expression of CKLF-like MARVEL transmembrane member 5 (CMTM5) by promoter methylation in myeloid leukemia. Leuk Res. 2011; 35:771-776.

10. Zhang W, Mendoza MC, Pei X, Ilter D, Mahoney SJ, Zhang Y, Ma D, Blenis J, Wang Y. Down-regulation of CMTM8 induces epithelial-to-mesenchymal transition-like changes via c-MET/extracellular signal-regulated kinase (ERK) signaling. J Biol Chem. 2012; 287:11850-11858.

11. Zhang Y, Wang R, Song H, Huang G, Yi J, Zheng Y, Wang J, Chen L. Methylation of multiple genes as a candidate biomarker in non-small cell lung cancer. Cancer Lett. 2011; 303:21-28.

12. Xie J, Yuan Y, Liu Z, Xiao Y, Zhang X, Qin C, Sheng Z, Xu T, Wang X. CMTM3 is frequently reduced in clear cell renal cell carcinoma and exhibits tumor suppressor activities. Clin Transl Oncol. 2014; 16:402-409.
13. Su Y, Lin Y, Zhang L, Liu B, Yuan W, Mo X, Wang X, Li $\mathrm{H}$, Xing X, Cheng X, Dong B, Hu Y, Du H, et al. CMTM3 inhibits cell migration and invasion and correlates with favorable prognosis in gastric cancer. Cancer Sci. 2014; 105:26-34.

14. Yuan W, Li T, Mo X, Wang X, Liu B, Wang W, Su Y, Xu L, Han W. Knockdown of CMTM3 promotes metastasis of gastric cancer via the STAT3/Twist1/EMT signaling pathway. Oncotarget. 2016; 7:29507-19. https://doi.org/10. 18632/oncotarget.8789.

15. Hu F, Yuan W, Wang X, Sheng Z, Yuan Y, Qin C, He C, $\mathrm{Xu}$ T. CMTM3 is reduced in prostate cancer and inhibits migration, invasion and growth of LNCaP cells. Clin Trans1 Oncol. 2015; 17:632-639.

16. Li Z, Xie J, Wu J, Li W, Nie L, Sun X, Tang A, Li X, Liu R, Mei H, Wang F, Wang Z, Gui Y, Cai Z. CMTM3 inhibits human testicular cancer cell growth through inducing cellcycle arrest and apoptosis. PLoS One. 2014; 9:e88965.

17. Li W, Zhang S. CKLF-Like MARVEL Transmembrane Domain-Containing Member 3 (CMTM3) Inhibits the Proliferation and Tumorigenisis in Hepatocellular Carcinoma Cells. Oncol Res. 2017; 25:285-293.

18. Barriere G, Tartary M, Rigaud M. Metformin: a rising star to fight the epithelial mesenchymal transition in oncology. Anticancer Agents Med Chem. 2013; 13:333-340.

19. Jiang W, Gu W, Qiu R, He S, Shen C, Wu Y, Zhang J, Zhou J, Guo Y, Wan D, Li Z, Deng J, Zeng L, et al. miRNA101 Suppresses Epithelial-to-Mesenchymal Transition by Targeting HMGA2 in Pancreatic Cancer Cells. Anticancer Agents Med Chem. 2016; 16:432-439.

20. Yang J, Mani SA, Donaher JL, Ramaswamy S, Itzykson RA, Come C, Savagner P, Gitelman I, Richardson A, Weinberg RA. Twist, a master regulator of morphogenesis, plays an essential role in tumor metastasis. Cell. 2004; 117:927-939.

21. Natarajan J, Chandrashekar C, Radhakrishnan R. Critical biomarkers of epithelial-mesenchymal transition in the head and neck cancers. J Cancer Res Ther. 2014; 10:512-518.

22. Mittal V. Epithelial Mesenchymal Transition in Aggressive Lung Cancers. Adv Exp Med Biol. 2016; 890:37-56.

23. Gao T, Li JZ, Lu Y, Zhang CY, Li Q, Mao J, Li LH. The mechanism between epithelial mesenchymal transition in breast cancer and hypoxia microenvironment. Biomed Pharmacother. 2016; 80:393-405.

24. Lin X, Xu W, Shao M, Fan Q, Wen G, Li C, Jing L, Sun X. Shenling Baizhu San supresses colitis associated colorectal cancer through inhibition of epithelial-mesenchymal transition and myeloid-derived suppressor infiltration. BMC Complement Altern Med. 2015; 15:126.

25. Feng J, Cen J, Li J, Zhao R, Zhu C, Wang Z, Xie J, Tang W. Histone deacetylase inhibitor valproic acid (VPA) promotes the epithelial mesenchymal transition of colorectal cancer cells via up regulation of Snail. Cell Adh Migr. 2015; 9:495-501. 
26. Delic S, Thuy A, Schulze M, Proescholdt MA, Dietrich P, Bosserhoff AK, Riemenschneider MJ. Systematic investigation of CMTM family genes suggests relevance to glioblastoma pathogenesis and CMTM1 and CMTM3 as priority targets. Genes Chromosomes Cancer. 2015; 54:433-443.

27. Wang Y, Li J, Cui Y, Li T, Ng KM, Geng H, Li H, Shu XS, Li H, Liu W, Luo B, Zhang Q, Mok TS, et al. CMTM3, located at the critical tumor suppressor locus 16q22.1, is silenced by $\mathrm{CpG}$ methylation in carcinomas and inhibits tumor cell growth through inducing apoptosis. Cancer Res. 2009; 69:5194-5201.

28. Abe M, Yamashita S, Mori Y, Abe T, Saijo H, Hoshi K, Ushijima T, Takato T. High-risk oral leukoplakia is associated with aberrant promoter methylation of multiple genes. BMC Cancer. 2016; 16:350.

29. Shen Z, Chen X, Li Q, Zhou C, Xu Y, Yu R, Ye H, Li J, Duan S. Elevated methylation of CMTM3 promoter in the male laryngeal squamous cell carcinoma patients. Clin Biochem. 2016; 49:1278-1282.

30. Yuan W, Liu B, Wang X, Li T, Xue H, Mo X, Yang S, Ding S, Han W. CMTM3 decreases EGFR expression and EGFmediated tumorigenicity by promoting Rab5 activity in gastric cancer. Cancer Lett. 2017; 386:77-86. 